Meta

Journal des traducteurs

Translators' Journal

\title{
Les cheminements de la créativité en traduction
}

\section{Bernd Stefanink et Ioana Bălăcescu}

Volume 60, numéro 3, décembre 2015

URI : https://id.erudit.org/iderudit/1036145ar

DOI : https://doi.org/10.7202/1036145ar

Aller au sommaire du numéro

\section{Éditeur(s)}

Les Presses de l’Université de Montréal

ISSN

0026-0452 (imprimé)

1492-1421 (numérique)

Découvrir la revue

Citer cet article

Stefanink, B. \& Bălăcescu, I. (2015). Les cheminements de la créativité en traduction. Meta, 60(3), 599-620. https://doi.org/10.7202/1036145ar

\section{Résumé de l'article}

La créativité est souvent sciemment écartée de la réflexion traductologique sous prétexte qu'elle " ne se prête pas à une étude systématique " (Gerzymisch-Arbogast et Mudersbach 1998: 16). Et pourtant elle est le pain quotidien du traducteur. Elle est donc incontournable dans une théorie qui tient compte de la pratique. Nous nous proposons de démontrer la pertinence de l'approche herméneutique dans la gestion de la créativité par un exemple empirique, étudié à l'aide de l'analyse conversationnelle ethnométhodologique, qui nous permet de suivre le cheminement vers une solution créative qui peut paraître osée à première vue, mais qui se voit brillamment légitimée par des recherches récentes en sciences cognitives sur le fonctionnement de notre cerveau.
Ce document est protégé par la loi sur le droit d'auteur. L'utilisation des services d'Érudit (y compris la reproduction) est assujettie à sa politique d'utilisation que vous pouvez consulter en ligne.

https://apropos.erudit.org/fr/usagers/politique-dutilisation/ 


\title{
Les cheminements de la créativité en traduction
}

\author{
BERND STEFANINK \\ Universidade Federal de Santa Catarina, Brésil \\ bstefanink@hotmail.com \\ IOANA BĂLĂCESCU \\ Universitatea din Craiova, Craiova, Roumanie \\ ioanadi@hotmail.com
}

\begin{abstract}
RÉSUMÉ
La créativité est souvent sciemment écartée de la réflexion traductologique sous prétexte qu'elle «ne se prête pas à une étude systématique» (Gerzymisch-Arbogast et Mudersbach 1998: 16). Et pourtant elle est le pain quotidien du traducteur. Elle est donc incontournable dans une théorie qui tient compte de la pratique. Nous nous proposons de démontrer la pertinence de l'approche herméneutique dans la gestion de la créativité par un exemple empirique, étudié à l'aide de l'analyse conversationnelle ethnométhodologique, qui nous permet de suivre le cheminement vers une solution créative qui peut paraître osée à première vue, mais qui se voit brillamment légitimée par des recherches récentes en sciences cognitives sur le fonctionnement de notre cerveau.
\end{abstract}

\begin{abstract}
Creativity is often deliberately pushed aside from translatological reflection under the pretext that it "does not lend itself to a systematic study" (Gerzymisch-Arbogast and Mudersbach 1998: 16). All the same, it is the daily bread of the translator. It is therefore essential in a theory that takes practice into account. We propose to demonstrate the relevance of the hermeneutic approach in the management of creativity with an empirical example using the ethnomethodological conversation analysis which allows us to witness the path that leads to a creative solution that might at first seem somewhat bold, but is brilliantly justified with the results of recent research in cognitive science showing how our brain really functions.
\end{abstract}

\section{MOTS CLÉS/ KEYWORDS}

créativité, herméneutique, recherches cognitives, analyse conversationnelle ethnométhodologique, barrières culturelles

creativity, hermeneutics, cognitive research, ethnomethodological conversation analysis, cultural barriers

La créativité est le moment de vérité, le moment existentiel de toute théorie de la traduction, qui doit se mesurer à la façon dont elle la gère.

(Stefanink: ci-dessous)

\section{Le courage de la créativité chez les praticiens}

Nous venons plaider la cause de la créativité en traduction, parce que nous avons pu constater que beaucoup d'étudiants arrivent à d'excellentes solutions créatives à un problème de traduction, mais les abandonnent au profit de solutions pâlottes par 
manque de moyens pour convaincre de la légitimité de ces solutions créatives, ne disposant pas des arguments susceptibles de les défendre. Ces arguments leur sont fournis par une réflexion théorique.

\section{La gestion de la créativité par les théoriciens}

Certaines approches traductologiques - appelons-les «analytiques» ou «objectivistes» - reconnaissent l'importance de l'intuition et de la créativité en traduction, mais «les excluent de leurs réflexions théoriques parce qu’elles ne sont pas systématisables»(Gerzymisch-Arbogast et Mudersbach 1998: 16). Nous prétendons, au contraire, que, si certains philosophes existentialistes ont pu parler de «moments privilégiés» (Sartre) ou de "moments existentiels» (Jaspers) dans lesquels se révèle l'essence de l'être, ce qu'Ernest Hemingway a pu appeler le «moment de vérité» en tauromachie, le «moment de vérité» d'une théorie en traduction, c'est celui où elle doit fournir une réponse à un problème soulevé par la traduction de l'imaginaire culturel. Ce moment de vérité est aussi celui de la créativité.

Dans l'exemple qui suit, nous nous proposons de montrer que l'approche herméneutique, appuyée par les recherches cognitivistes récentes sur le fonctionnement du cerveau humain, répond parfaitement à cette exigence. Avant de passer à cet exemple, nous nous permettons de rappeler certains aspects théoriques concernant, d'une part, la démarche herméneutique, et, d'autre part, les recherches cognitivistes récentes qui viennent la confirmer.

\section{Tout est une question épistémologique d'accès au sens}

Pour les tenants des approches analytiques, le sens est dans le texte et doit être décortiqué peu à peu au cours d'une "analyse-du-texte-pertinente-pour-le-traducteur» [übersetzerrelevante Textanalyse] (Hönig 1989) avant même toute tentative de traduction à proprement parler. Pour les herméneutes, au contraire, le sens se construit dans un «dialogue avec le texte» qui mène à une «fusion des horizons" [Horizontverschmelzung] entre l'horizon du texte et l'horizon du lecteur. Fusion intime qui finit par aboutir à ce que Radegundis Stolze (2003: 216) a appelé « une impulsion de formulation autopoḯtique intuitive semi-consciente» en langue cible. C'est là le lieu privilégié pour la créativité. Cette approche herméneutique est soutenue par les recherches récentes en sciences cognitives sur le fonctionnement du cerveau humain, notamment sur le rôle de la subjectivité, d'une part, et de la métaphore, d'autre part, dans la construction du sens.

\section{L'approche herméneutique légitimée par les découvertes récentes des cognitivistes et des neurophysiologistes}

Malgré les noms de grands philosophes herméneutes, comme Paul Ricœur ou Martin Heidegger, ou encore de Hans-Georg Gadamer, on n'a eu très longtemps que des sourires condescendants pour les traductologues qui ont pu s'approprier ces théories pour les appliquer à l'opération traduisante. On a pu trouver l'approche herméneutique «préscientifique», la taxant de «mysticisme». Ceci notamment en raison de sa terminologie métaphorique. Ce que nous avons appelé la «chasse au fantôme de 
l'objectivité» (Stefanink 1997) était trop fortement ancré dans les esprits des traductologues. La méthode pour arriver à une traduction dite «objective» était basée sur une dissection du texte source en éléments de sens dits pertinents pour la compréhension (Gerzymisch-Arbogast et Mudersbach 1998: 47).

Or, des chercheurs cognitivistes comme Lakoff et Johnson (1980) ont dénoncé ce qu'ils ont appelé «The Myth of Objectivism in Western Philosophy», mythe auquel ils consacrent un chapitre entier dans un livre qui porte le titre de Metaphors We Live by. Livre dans lequel non seulement ils dénoncent ce mythe mais revalorisent aussi la métaphore - caractéristique de la terminologie herméneutique - comme instrument d'accès au sens. En effet, dans un autre livre qui porte le titre fascinant et prometteur de Women, Fire and Dangerous Things, Lakoff (1987) a démontré ce que les philosophes herméneutes - notamment Martin Heidegger dans Sein und Zeit (1927) - ont décrit par le "cercle herméneutique», à savoir l'incontournable caractère subjectif de nos perceptions.

George Lakoff nous rappelle que notre processus de compréhension passe par une catégorisation: "Without the ability to categorize, we could not function at all» (1987: 6). Il continue, en précisant que cette catégorisation est le résultat de nos expériences, de notre culture, de notre perception et de notre activité motrice (1987: 8). On sait, par ailleurs, que la condition sine qua non de la catégorisation est le caractère récurrent de ces expériences.

On ne peut pas alors s'empêcher de faire le rapprochement avec ce que les neurophysiologistes nous apprennent sur la gestion des informations qui parviennent à notre cerveau, à savoir que des informations qui se ressemblent (Wittgenstein parlerait de Familienähnlichkeit ${ }^{1}$ ) ont tendance à emprunter les mêmes voies neuronales. Ceci entraîne un processus de réciprocité au cours duquel certaines voies neuronales se renforcent au détriment d'autres, dans la mesure où les expériences récurrentes se regroupent pour emprunter, par attraction, ces voies neuronales privilégiées qui, ainsi renforcées, exercent une force d'attraction encore plus grande sur des expériences du même type. Notons à cet endroit le parallélisme avec l'insistance des cognitivistes sur le fait que les expériences récurrentes qui mènent à la catégorisation doivent être du même type, kind chez Lakoff (1987: 5; italiques dans l'original): «Every time we see something as a kind of thing, for example a tree, we are categorizing.» La base matérielle de cette catégorisation est évidemment la voie neuronale privilégiée, constituée en engrammes et attirant à elle toutes les informations du même kind.

Cette conception est confirmée par des chercheurs connexionnistes qui constatent que ce n'est pas la qualité de l'information qui est responsable de la force d'une voie neuronale, mais la fréquence (à ce propos, les neurophysiologistes parlent de firing) avec laquelle elle est sollicitée (Schade 1992: 39).

Des neurophysiologistes, comme Pritzel, Brand et al. (voir 2003: 39-96), nous montrent comment ces réseaux neuronaux se constituent matériellement. Il s'agit d'un processus d'apprentissage effectué par les chaînes neuronales à partir de nos expériences récurrentes. Examinons ce processus.

À part son noyau, un neurone est constitué d'une partie réceptrice d'information et d'une partie transmettrice d'information. La partie réceptrice est constituée de ramifications appelées dendrites, sur lesquelles poussent des épines, sensibles aux excitations venant de l'extérieur. La partie transmettrice est constituée par un axone, qui entre en contact avec les synapses qui constituent la zone de contact entre les 
cellules nerveuses. Le nombre d'épines et de ramifications dans la partie réceptrice va croissant en fonction de la fréquence des excitations reçues. À l'opposé, des voies neuronales peu utilisées voient leurs synapses s'étioler et mourir, contribuant ainsi à la formation de voies neuronales renforcées par rapport à d'autres, c'est-à-dire les engrammes (en allemand Gedächtnisspuren ${ }^{2}$ ).

Par ailleurs, les neurophysiologistes constatent que les informations stockées dans notre cerveau sous forme d'engrammes ne le sont pas de façon isolée mais sont reliées entre elles de façon associative. On peut donc parler de réseaux engrammatiques qui structurent notre mémoire et à travers lesquels nous saisissons la réalité, y compris celle d'un texte. C'est, là encore, une constatation qui recoupe et confirme les observations de Lakoff et Johnson, pour lesquels «much of our conceptual system is structured by metaphor» $(1980$ : 147) et «our conceptual system is entirely metaphorical» (1980: 184). Lakoff et Johnson parlent de «metaphorical entailments» et ils écrivent à ce propos: «[...] understanding takes place in terms of entire domains of experience and not in terms of isolated concepts» (1980:117), puis «[...] conceptual metaphors are grounded in correlations within our experience» (1980: 154-155).

Ce sont ces corrélations métaphoriques qui sont à la base de notre créativité. Lakoff constate que dans la métaphorique populaire (il appelle cela des folk models of categorization) telle qu'elle se reflète dans des tournures métaphoriques - d'une part, pour exprimer la haine, comme «He was breathing fire. Those are inflammatory remarks. She was doing a slow burn. Your insincere apology just added fuel to the fire. After the argument, Dave was smoldering for days » (Lakoff, 1987: 388) et d'autre part, pour au contraire exprimer l'amour, comme "She is an old flame. Hey baby light my fire. She's hot stuff. I've got the hot's for her» (Lakoff, 1987: 410) - on assiste à une association entre les deux catégories «haine» (avec le scénario prototypique anger is fire) et «désir» (avec le scénario prototypique lust is heat) au moyen d'un noyau central de cette catégorie qui est le "feu», ce qui correspond bien aux principes de la sémantique des prototypes d'Eleanor Rosch (1973), selon lesquels, dans la pratique du vécu, les délimitations entre les catégories ne sont pas aussi clairement définies que le voulait la sémantique structurale. Dans sa pratique quotidienne, l'être humain saute d'une catégorie sémantique à l'autre par association. C'est là exactement ce que font les traducteurs de nos corpus de données lorsqu'ils sont à la recherche d'une solution à un problème de traduction suscité par des différences d'ordre culturel.

Ainsi la créativité devient une nécessité pour le traducteur du fait que les systèmes conceptuels, d'ordre métaphorique, qui structurent notre perception de la réalité, "are grounded in our experience», comme disent Lakoff et Johnson, et que ces « conceptual systems [...] vary from culture to culture» (1980: 146), contraignant ainsi le traducteur à la créativité, s'il veut passer d'un système culturel à l'autre avec l'intention de produire le même effet et obtenir la Wirkungsgleicheit [adéquation de l'effet produit] préconisée par Reiß et Vermeer (1974).

Parallèlement, le philosophe Hans Lenk a poussé plus loin ces recherches sur les rapports entre ces systèmes conceptuels basés sur notre expérience et les découvertes récentes des neurophysiologistes. Se référant aux recherches de Hubel et Wiesel, il introduit la notion de "schéma» pour désigner le cadre engrammatique à travers lequel nous saisissons la réalité, le «schéma» rappelle la fonction des catégories chez Lakoff. Il se constitue au fil de nos expériences vécues et est par conséquent de nature 
subjective. Hubel et Wiesel ont constaté que nous n'avons pas une saisie immédiate d'un objet tel quel, mais que les neurones qui sont activés par la vue de cet objet sont localisés à des endroits différents du cerveau, ce qui laisse penser qu'il y a des neurones spécialisés qui vont saisir, par exemple, les dimensions de l'objet, alors que d'autres vont en saisir la couleur. Le cerveau procède, en effet, dans un premier temps, à une analyse et décomposition, pour ensuite aboutir à une synthèse à un niveau supérieur où l'image intégrale sera reconstruite. Nous saisissons donc la réalité à travers des ensembles neuronaux, des neuronal assemblies (Lenk, 2014: 78), qui constituent la base du «schéma». Un schéma qui, à la différence de celui que Kant avait introduit dans la discussion scientifique, n'est pas une catégorie fixe et univoque de l'esprit, la même pour chaque être doué de raison, mais une catégorie flexibilisée et libéralisée, imprégnée [imprägniert] (Lenk, 2014: 81) par la réalité qu’elle saisit, c'est-à-dire qu'à chaque nouvelle sollicitation par un objet réel, l'ensemble neuronal, support matériel du "schéma" correspondant, est activé et renforcé - les neurones activés se mettant au diapason de l'ensemble neuronal déjà existant ${ }^{3}$-, mais est également imprégné d'une nouvelle nuance. Ces ensembles neuronaux constituent la base physiologique des réseaux associatifs (les folk models of categorization lakoffiens que nous venons de voir ci-dessus) qui interviennent dans la construction du sens qui se construit dans la psyché du traducteur au contact du texte.

La construction de ce sens se fait dans un processus au cours duquel interagissent, dans un mouvement conjoint, les éléments linguistiques du texte, ce que les cognitivistes appellent le processus bottom-up et les éléments d'expériences stockées dans la mémoire du récepteur du texte, ce que les cognitivistes appellent le processus top-down. Ne retrouvons-nous pas là la fusion des horizons des herméneutes, la Horizontverschmelzung gadamérienne, que les «objectivistes» ont tellement tournée en dérision?

Quant à la subjectivité incontournable du traducteur, que l'approche herméneutique a intégrée dans sa réflexion théorique et qui met mal à l'aise les défenseurs des approches analytiques, elle trouve ses assises dans ce que nous avons appelé plus haut des réseaux engrammatiques qui constituent en fait la base matérielle des schemata tels que les conçoit Hans Lenk. Lorsque Lenk écrit: «Wir haben keinen interpretationsunabhängigen Zugang zur Welt» [Nous n'avons pas un accès au monde indépendant de notre interprétation] (Lenk, 2014: 71), il s'empresse de préciser que l'incontournable caractère interprétatif de cet accès au monde ne doit pas être conçu comme une pure projection de l'esprit, idéaliste et subjective, mais qu'il s'agit d'une interprétation active, d'une Spezialform des interaktiven Interpretierens (Lenk 2014: 81) qu'il appelle imprégnation [Imprägnieren] (Lenk 2014: 81):

«L'imprégnation» telle que nous la concevons est en fait quelque chose qui relie l'apport du monde réel à un processus structurant dans lequel nous 'entrons' nous-mêmes; et les deux sont imbriqués l'un dans l'autre. (Lenk 2014: 81; notre traduction) ${ }^{4}$

En termes herméneutiques, nous retrouvons là un «dialogue avec le monde» qui n'est pas sans rappeler le «dialogue avec le texte» gadamérien, venant ainsi, là encore, soutenir la pertinence de l'approche herméneutique en traduction. 


\subsection{Les observations des herméneutes, des cognitivistes, des connexionnistes et des neurophysiologistes se concrétisent dans la pratique du traducteur}

Toutes ces observations des herméneutes, des cognitivistes, des connexionnistes et des neurophysiologistes sont confirmées par la pratique du traducteur. Ainsi, dans son auto-témoignage sur son activité traductive, Irina Mavrodin, praticienne qui a été décorée de l'ordre de "Chevalier des Arts et Lettres» par le gouvernement français, pour la qualité de ses traductions, parle de lanțul semnificant (1982: 132), c'està-dire de l'enchaînement significatif, et du mécanisme associatif (1982: 137) qui déterminent sa compréhension du texte à traduire et ses choix traduisants. De même, les corpus que nous a fournis l'analyse conversationnelle ethnométhodologique viennent largement confirmer cet autotémoignage: les enchaînements associatifs, qu'on les appelle metaphorical entailments, lanțuri semnificative ou chainings, sont source de créativité traductive.

Nous voyons donc la pertinence de l'approche herméneutique démontrée aux deux extrêmes: celui du praticien, d'une part, qui aborde la traduction dans ce qu'il appelle le " "dialogue" avec le texte ${ }^{5}$ et, d'autre part, celui du chercheur en sciences cognitivistes, qui apporte la légitimation scientifique du terme gadamérien de la «fusion des horizons».

\section{Nécessité d'un nouveau critère d'évaluation}

Mais où donc réside, après tout ce que nous venons de dire, le critère d'évaluation d'une traduction, puisque "l'analyse-du-texte-pertinente-pour-le-traducteur», garante présumée d'objectivité dans les approches analytiques, n'est plus? Là encore les recherches cognitivistes sur la créativité nous apportent la réponse. Les chercheurs en créativité ont déterminé les conditions sine qua non dans lesquelles on peut parler de créativité. Pour qu'un event (au sens où l'entend la philosophie analytique) soit considéré comme créatif, il doit répondre à deux critères: 1) il doit être inédit; et 2) il doit satisfaire au critère d'acceptation, qui correspond au critère poppérien de la reconstruction rationnelle du chemin parcouru (Popper 1935), sur lequel nous reviendrons.

Pour satisfaire à ce critère d'acceptation, nous utilisons le terme Intersubjektive Nachvollziehbarkeit (Stefanink 1997), qu'on a pu traduire par plausibilité intersubjective bien que le mot plausibilité, de nature statique, ne rende pas tout à fait le caractère dynamique et procédural qu'implique le mot Nachvollziehbarkeit, ce dernier entendant une implication «corps et âme» dans le processus de compréhension ${ }^{6}$; une traduction par traçabilité intersubjective semble mieux rendre cette dynamique.

Le traducteur herméneute ne doit pas donner libre cours à une créativité débridée, sur les traces de l'École de la manipulation (Hermans, 1985) pour laquelle le traducteur est seul juge de ce qu'il veut appeler une traduction. La déontologie du traducteur herméneute lui intime de légitimer sa traduction aux yeux d'autrui et ceci plus particulièrement lorsqu'il est parvenu intuitivement à une solution créative à un problème de traduction. Dans l'exemple qui suit, nous nous proposons de fournir une telle légitimation à une traduction créative qui peut paraître très osée et d'illustrer la construction du sens dans une approche herméneutique à l'aide de ce cas concret. 


\section{Exemple de légitimation d'une solution créative trouvée dans une approche herméneutique}

À cette fin, nous allons analyser la démarche de deux informateurs en train de «négocier » la solution d'un problème de traduction. Nous allons examiner le cheminement de leurs «enchaînements associatifs » et essayer de les expliquer par des approches cognitivistes.

Le texte à traduire était tiré du Canard enchaîné et se situe en $1983^{7}$, alors que le gouvernement socialiste de François Mitterrand était en place depuis deux années. Deux années pendant lesquelles les socialistes ont été beaucoup critiqués, jusque dans leur absence de présence médiatique (la mesquinerie allait, par exemple, jusqu'à leur reprocher de ne pas savoir s'habiller).

Comme on pouvait s'y attendre, Le Canard enchaîné, hebdomadaire satirique, critique du gouvernement, se faisait une fête d'exploiter ces maladresses. Tout au long de notre texte, il compare les ministres à une salle de classe d'élèves indisciplinés, qui ne pensent qu'à se moquer de leur président, qui a toutes les caractéristiques d'un instituteur décidé à faire régner l'ordre dans sa classe. Le coup fatal est porté au dernier paragraphe. Si, jusque-là, le lecteur avait pu penser à un jeu innocent, où l'on se permet de petites pointes à l'égard du gouvernement, la dernière phrase donne tout à coup à ces remarques (qui paraissaient lancées au hasard, au gré de leur apparition dans l'imaginaire de l'auteur) une cohérence révélatrice de la fonction globale du texte: «L'heure du casse-croûte, c'est sacré!» Voyons ce texte:

Le mercredi, à l’Élysée RÉUNION DU CONSEIL DE DISCIPLINE

Avant le dernier remaniement, ils étaient trente-six (en comptant Mitterrand) autour de la table du Conseil des ministres. Ils ne sont plus aujourd'hui que dix-sept. La table étant restée la même, ces messieurs-dames ont pu se desserrer.

Mais à entendre certains d'entre eux, cette aération comporte des inconvénients. Les discussions en aparté sont désormais à peu près impossibles et, lorsqu'un ministre intervient, il devient très risqué d'échanger en douce quelques vacheries à son endroit. L'œil noir de Tonton fusille immédiatement le bavard. Alain Savary, dont c'était un des sports favoris jusqu'à cette regrettable petite révolution, est, paraît-il, terriblement frustré. La classe étant moins nombreuse, et plus facile à surveiller, il est devenu quasiment impossible de lire tranquillement son journal ou de faire son courrier sans se faire repérer illico.

Autre petit jeu pratiquement refusé désormais aux ministres: la rédaction des petits mots que, traditionnellement, ils se passent de l'un à l'autre pour se distraire au cours des exposés parfois barbants de leurs distingués collègues.

Et puis, le brouhaha est proscrit. Tonton a voulu imprimer un style nouveau au Conseil. Maintenant, il donne systématiquement la parole à tous ceux qui la demandent, et il insiste même pour avoir l'avis des principaux ministres sur tel ou tel sujet. Ce qui fournit, paraît-il, l'occasion à Michel Rocard de faire de véritables exposés dans le style Sciences-Po.

Enfin, le Conseil doit être désormais terminé à $12 \mathrm{~h} 30$ pétantes. Plus question de jouer les prolongations. L'heure du casse-croûte, c'est sacré!

(Krings, 1986: 87; Stefanink, 1991: 82). 


\section{La recherche des équivalences, une solution?}

Le traductologue Krings (1986) a utilisé ce texte pour étudier les démarches suivies par ses informateurs (étudiants allemands de français, en quatrième année d'études) pour traduire ce texte en allemand, ceci en procédant à une analyse de leurs protocoles de verbalisation (think aloud protocols). La dernière phrase du texte leur a posé problème. C'est surtout le mot casse-croûte, culturellement marqué et n'ayant pas de correspondant en allemand, qui a été l'objet de leurs préoccupations. Partant d'une conception de la traduction dominée par le principe de la recherche des équivalences, Krings a fait la même erreur que certains de nos informateurs, qui se sont acharnés à vouloir trouver un mot qui corresponde à casse-croûte sans comprendre que, comme pour les couleurs dans l'œuvre de Proust, c'est la valeur symbolique de ce mot qui est importante dans ce texte et que c'est cette valeur symbolique qu'il faut traduire.

Krings, encore attaché aux principes de la démarche analytique, a trouvé le mot allemand Stulle pour rendre casse-croûte. Le fait que pas un seul de ses informateurs n'ait eu ce mot à la bouche tout au long de sa recherche (tout comme d'ailleurs nos propres informateurs) montre bien à quel point ce mot est peu répandu. Il est indiqué par les dictionnaires comme diatopiquement marqué et connu uniquement dans le nord de l'Allemagne. Et là notre expérience personnelle nous dit qu'il est connoté avec une tranche de pain de seigle sur laquelle on a chichement gratté un peu de margarine, agrémenté d'une fine tranche de saucisson en filigrane. Le tout pris dans la pénombre d'un repas de famille silencieux où l'on se parle juste pour demander le sel. Rien à voir avec la baguette sandwich jambon fromage, d'où le camembert déborde des deux côtés sous les crocs avides de l'ouvrier en bâtiment français plein de vie.

D'autres informateurs ont également cherché à se concentrer sur la consistance matérielle du casse-croûte, cherchant des équivalents du côté du paradigme des petits repas pris à la sauvette, comme snack, sandwich ou Imbiss: il s'agit d'une recherche relevant de la théorie des équivalences basée, dans notre cas, sur le trait pertinent de la taille du repas. Cette focalisation «atomisante» sur la traduction d'un mot empêche de voir le vrai sens que prend ce mot dans ce texte. Vu en termes cognitivistes, les premiers se sont trop concentrés sur les éléments bottom-up, essayant d'analyser en traits pertinents la nature du casse-croûte, les seconds font appel à leur bagage cognitif, leur savoir au quotidien, donc au processus top-down, l'expérience leur ayant appris que - comme le dit une informatrice roumaine dans un autre corpus conversationnel concernant la traduction de ce même texte: «mais... à midi, c'est pas le casse-croûte!? » et traduisent par Mittagessen [repas de midi].

\section{L'approche holistique et la fusion des horizons (Horizontverschmelzung; Gadamer, 1960) chez le traducteur herméneute}

Le traducteur herméneute, quant à lui, aborde la question sous un angle holistique. Il se demande quelle est la fonction de ce mot dans le texte, pourquoi l'auteur a-t-il choisi, dans le paradigme des types de repas, un élément qui sort de la norme, donc est particulièrement informatif et correspond à une intention de l'auteur.

Il est sensibilisé à l'imaginaire culturel véhiculé par le mot casse-croûte, par ce que le cognitiviste Roger Schank (1982) appelle le script. Si l'on en croit la théorie du script, le lecteur ne vient pas naïvement à ce texte! En achetant Le Canard enchaîné, il a certaines attentes qui font partie du script dans sa tête: il sait qu'à cette 
époque, deux ans après l'élection d'un gouvernement socialiste avec François Mitterrand à sa tête, ce journal est critique de ce gouvernement. Si, en outre, il a vécu à cette époque en France, il connaît ce que les herméneutes appellent l'horizon $d u$ texte. Cet horizon était marqué, de la part de la droite politique, par un profond mépris pour les façons - disons "prolétaires» - maladroites que les socialistes au pouvoir avaient de se comporter.

C'est à travers ce script qu'il faut lire le mot casse-croûte dans ce texte. Le mot est ici emblématique de la condition prolétaire. Cette fonction emblématique est renforcée par le contexte immédiat où le mot prolongations est tiré du vocabulaire du football. Or, dans l'imaginaire culturel des Français, le football est connoté avec le vulgaire prolétaire ${ }^{8}$ Même vulgarité prolétarienne en ce qui concerne le mot pétantes, là encore choisi à dessein - donc porteur d'information voulue par l'auteur dans le paradigme des mots indiquant la précision dans le temps, au lieu de « $12 \mathrm{~h} 30$ heures précises». Et pour couronner le tout: irruption du langage parlé, avec la rupture du rythme de la phrase, dans un texte qui par ailleurs appartient au bon style littéraire. Sous-entendu: le prolétaire ne sait pas formuler des phrases bien construites! C'est là encore un choix syntaxique délibéré de la part de l'auteur qui vient soutenir ce qu'il a exprimé par le choix des lexèmes.

On voit donc comment le sens du mot casse-croûte se construit dans une fusion de l'horizon du texte (processus bottom-up), représenté par les éléments linguistiques que nous venons de citer, avec l'horizon du lecteur (top-down) représenté par les éléments du script que véhicule sa psyché.

Mais le script du lecteur ne se limite pas à cela. Le scénario du prolétariat, représenté par le mot casse-croûte et l'imaginaire culturel qu'il véhicule, fait intervenir un deuxième script, auquel l'auteur du texte fait clairement appel dans la mémoire collective et dans l'imaginaire collectif des Français: un ouvrier n'a pas le sens des responsabilités! Il est habitué à des horaires fixes, parce qu'il fait un travail à la chaîne dans lequel il n'est pas responsabilisé. Traduisez: le gouvernement socialiste n'a pas le sens des responsabilités, sous-entendu: contrairement au gouvernement de droite. C'est cela l'information véhiculée par notre texte! C'est là le rhème de notre texte!

\section{L'insatisfaction, point de départ de la créativité}

Après avoir ainsi analysé le processus de compréhension, tournons-nous vers le processus de production (étant entendu que, dans l'opération traductive, les deux processus ne sont pas séparables, même si, dans ce cadre, pour des raisons d'analyse et de présentation didactique, ils sont traités séparément). Là encore, la prise en considération des processus mentaux mis en évidence par les chercheurs cognitivistes, permet de mieux comprendre la démarche traductive mise en ouvre.

Les informateurs de Krings ont trouvé comme solution Die Stunde des Mittagessens ist heilig [L'heure du repas de midi est sacrée], une solution relativement pâlotte, à caractère informatif, qui n'est guère compatible avec le caractère émotionnel de cette phrase, émotivité qui s'exprime dans le fait de reprendre à son compte un parler oral vivant qui pourrait être celui du prolétaire dans cette situation. Dans la formulation française, on voit un de ces ministres littéralement prononcer cette phrase, elle est "vivante», alors que dans la traduction allemande, on voit l'auteur en train d'informer, de commenter et d'expliquer. La traduction allemande manque de 
dynamisme. Or, Le Canard enchaîné paraît une fois par semaine et ne peut avoir pour caractéristique principale d'informer, puisque le lecteur aura eu les informations par d'autres médias bien avant. Cette traduction reste donc insatisfaisante par rapport à ce que veut exprimer l'original.

Nos premières tentatives de traduction, qui ont abouti comme celles de beaucoup d'autres informateurs à la solution Die Mittagspause ist heilig [La pause de midi est sacrée] nous ont laissés insatisfaits. Cette insatisfaction - l'insatisfaction, selon les chercheurs en créativité, est, ne l'oublions pas, à la base de toute créativité - nous a amenés à nous replonger dans le texte, fidèles aux principes d'un Paepcke (Paepcke et Forget 1981), d'après lequel la traduction est «ascèse intellectuelle» et poussée par ce «besoin immédiat» de «l'effort quotidien» qui selon Einstein (cité par Mavrodin

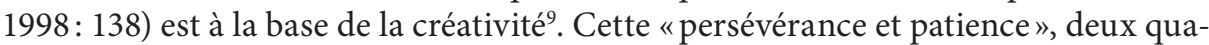
lités fondamentales, selon Einstein, de l'être créatif, qualités que, selon Mavrodin (voir 2001: 49), doit posséder chaque traducteur, nous ont amenés peu à peu à une intimité et à une empathie croissante avec le texte, jusqu'à aboutir à cette Horizontverschmelzung gadamerienne (le contopire chez Mavrodin, 1998: 137, qui lui préfère toutefois le terme impersonnalisation), qui a amené la solution créative suivante, sous l'effet de ce que Stolze (2003) a appelé une impulsion de formulation intuitive [Intuitiver Formulierungsimpuls] : Wenn es schellt, wird der Ranzen gepackt! [Quand ça sonne, on range le cartable]; (il faut noter que la phrase allemande est à la voix passive, avec une fonction impérative, ce qui renforce le caractère incisif, péremptoire et «cassant» de la formulation).

\section{La créativité entre "mysticisme" et "problem solving activity" (Guilford 1975) au sein d'une traductologie qui se situe entre «art ou science» (Ionescu 2003)}

Lorsque, dans les années 1990, Stefanink a introduit progressivement l'étude de la créativité dans ses cours de traduction à l'Université de Bielefeld, il a eu droit aux ricanements de certains collègues: "Comment veux-tu enseigner la créativité? La créativité est un don, un art, ça ne s'enseigne pas!» Opinion qui est aussi répandue dans les milieux traductologiques, si l'on en croit Gerzymisch-Arbogast et Mudersbach (1998) qui certes ne nient pas l'importance de l'intuition et de la créativité dans l'opération traduisante, mais l'excluent de leurs réflexions en raison de leur caractère non systématisable ${ }^{10}$. Il n'est évidemment pas question de fournir une démarche algorithmique conduisant directement à des solutions créatives. Mais, d'un point de vue didactique, il y a fort à faire pour informer l'apprenant sur les processus cognitifs éclairant le fonctionnement de la créativité, comme nous le décrivent les chercheurs en créativité. Le but didactique est d'apprendre à créer des situations favorables à l'éclosion de la créativité!

\section{Un critère de scientificité: la reconstruction rationnelle du chemin qui a mené à la solution créative (Popper 1935)}

D'après Karl Popper, rien ne peut expliquer la première phase d'une «invention », elle relève du domaine de l'art. Popper (1935) distingue cependant une deuxième phase qui est celle de l'application-test de cette invention et de la reconstruction ration- 
nelle du chemin parcouru pour y arriver, reconstruction qui ne fournira toutefois jamais qu'un squelette, qui ne permettra jamais de programmer des inventions.

\section{Les critères de Popper appliqués à l'approche herméneutique: «Intersubjektive Nachvollziehbarkeit » comme base d'évaluation}

Si, dans les sciences exactes, une deuxième phase légitime l'invention et détermine son acceptation - l'appropriateness, des chercheurs en créativité - par la société au vu des résultats de son application, le traducteur, quant à lui, n'a pas de preuves matérielles du fonctionnement de son "invention», il doit les chercher dans ce que nous appelons la intersubjektive Nachvollziehbarkeit qui constitue la nouvelle base d'évaluation de la traduction, contribuant ainsi à ce que Cercel (2013: 122) a appelé la Verwissenschaftlichung der Übersetzungshermeneutik [l'accès au statut de science de l'herméneutique en traduction].

C'est à une telle reconstruction rationnelle que nous nous proposons de procéder, à titre exemplaire et didactique, en nous basant sur notre corpus conversationnel comme banque de données et en nous servant de l'analyse conversationnelle ethnométhodologique comme méthode de recherche, le processus par lequel nous sommes arrivés à ce résultat devenant plus important que le résultat lui-même, conformément aux réflexions d'un Gide ou d'un Valéry.

\section{Une méthode de recherche : «l'ethnotraductologie» (Stefanink 1995)}

L'analyse conversationnelle ethnométhodologique remonte aux années 1970, quand les chercheurs en sociologie ont développé cette méthode pour étudier les idées naïves que le commun des mortels non spécialisé véhicule à propos d'une science (Garfinkel 1984). Ils ont donné le nom d'«ethnoscience » à ce type de recherches. Ainsi, l'« ethnomédecine» étudie l'imaginaire véhiculé par les «hommes en blanc» et toutes les idées préconçues afférant à la médecine. Nous nous sommes approprié cette méthode pour l'appliquer aux recherches sur les idées naïves véhiculées par nos étudiants sur la traduction et tout ce qui y affère, à savoir leurs idées sur la langue, sur les éléments d'un texte, sur l'opération traductive, sur ce qu'ils ont appelé «l'atmosphère d'un texte», sur la place et le rôle des éléments culturels dans l'opération traductive, etc. Stefanink (1995) a appelé cette méthode de recherches l'« ethnotraductologie».

L'analyse conversationnelle ethnométhodologique appliquée à la traduction consiste à placer deux à quatre informateurs en face d'un texte dont ils doivent «négocier» une version commune en langue cible. Cette négociation permet à l'enseignant de dégager les manques à gagner sur le plan didactique, fournissant ainsi les bases de la structuration de son cours, voire d'un cursus de traduction, basé sur les problèmes abordés au cours de la négociation. Elle permet aux apprenants, d'une part, de prendre conscience (sous la houlette de l'enseignant) des idées d'ordre théorique qu'ils véhiculent inconsciemment, ce qui les rend d'autant plus réceptifs à l'enseignement du cadre théorique dans lequel viennent s'intégrer leurs idées naïves - conformément à notre thèse qu'aucune tête de traducteur n'est vierge de théorie (Bălăcescu et Stefanink 2003) - , et, d'autre part, de déclencher le mécanisme des chaînes associatives qui sont à la base de la créativité, comme nous l'enseignent les chercheurs cognitivistes en créativité. 
C'est à la suite de l'insatisfaction ressentie par les solutions qu'il avait trouvées au problème de traduction qui se posait que Stefanink a proposé à Bălăcescu de devenir sa partenaire dans la négociation de cette traduction. Cette négociation a abouti à une solution créative surprenante: la traduction de «L'heure du casse-croûte, c'est sacré!» par Wenn es schellt, wird der Ranzen gepackt![lorsque ça sonne, le cartable est rangél. Voyons ce que nous apprend le corpus de cette négociation. Appelons-le «corpus créatif» (voir annexe).

\section{Quels sont les processus cognitifs qui ont permis d'aboutir à cette solution créative?}

a) La visualisation de scènes

Commençons par constater que notre compréhension passe bien par un processus de visualisation, comme nous le décrit Fillmore (1976) dans sa sémantique des scenes-and-frames. Notre corpus nous fournit des indicateurs explicites de ce processus de visualisation avec ich sehe dann gleich Szenen [je vois tout de suite des scènes] et sich vorstellen [se représenter] qui introduisent la description de la scène de la classe d'élèves nonchalants, ennuyés par ce que dit le maître, attendant la pause.

b) L'énumération des détails scéniques

L'énumération des détails de cette scène est un des éléments qui amènera la solution. L'informateur A laisse planer son regard intérieur sur la salle de classe avec ses traits caractéristiques: l'ennui (gelangweilt), le maître qui parle dans le vide (ohne richtiges Interesse, herumstottert), les élèves attendant l'heure de la pause..., etc.

c) Le choix d'un élément proéminent dans la scène

Puis, dans cet amas de choses, un élément proéminent: le cartable, le Ranzen...

d) Une impulsion de formulation intuitive (Stolze 2003)

Elle impose à l'informateur A la formulation créative: Wenn es schellt, wird der Ranzen gepackt.

e) Un facteur déterminant: la structure rythmique

Jusque-là nous avons assisté à une démarche qui caractérise fondamentalement la majorité des cas qui ont mené à des solutions créatives. Dans le cas particulier de notre corpus, un élément supplémentaire a pris de l'importance: le rythme. C'est le mot knackig [qui fait un bruit sec] qui est révélateur. Il évoque en général quelque chose de cassant, mais aussi quelque chose de péremptoire et de tranchant, lorsqu'il se rapporte à la voix d'une personne ou à son comportement. Le mot entschieden [déterminé], par lequel l'informateur A caractérise également la dernière phrase du texte français et sa traduction allemande, vient soutenir et renforcer le knackig. Ce qui donne cette impression de quelque chose de cassant, de péremptoire et de tranchant, c'est là le rythme de cette dernière phrase. La phrase française a quelque chose de catégorique que nous retrouvons dans cette traduction allemande. Même l'informatrice 1 de notre corpus allemand ${ }^{11}$, qui, par ailleurs, ne brille certainement pas par sa sensibilité aux caractéristiques stylistiques du texte, montre qu'elle a senti cet aspect catégorique lorsqu'elle commente: «même si une discussion est intéressante 
au point que ça puisse durer plus longtemps, il n'en est pas question, ça ne se discute pas: schluss ist schluss! casse-croûte ist casse-croûte!»

Ces deux derniers bouts de phrase sont particulièrement révélateurs de l'importance du rythme. L'informatrice 1 du corpus allemand glose cette dernière phrase de notre texte par un phraséologisme - schluss ist schluss! [fini c'est fini!] - qui tire son sens principalement du rythme. En effet, il s'agit d'une formulation tautologique, sans aucune valeur informative du point de vue du sens véhiculé par les mots: le sujet et le prédicat sont identiques. La seule valeur informative vient du rythme qui exprime quelque chose de catégorique. Le renforcement par casse-croûte ist cassecroûte! est du même ordre: il n'informe pas par la sémantique des mots, mais tire toute sa valeur informative du rythme.

L'importance du rythme de cette phrase dans l'ensemble du texte est soulignée par le fait que l'informatrice $\mathrm{B}$ de notre corpus créatif est immédiatement d'accord avec la traduction proposée par A - Wenn es schellt, wird der Ranzen gepackt! - bien qu'elle ne comprenne pas le mot Ranzen, ce qui montre bien qu'elle aussi a surtout été sensible au rythme qui est le même dans cette phrase et la traduction allemande ${ }^{12}$ (précisons que le locuteur fait une pause après schellt et que cette première partie de la phrase se prononce avec une intonation montante, pour retomber ensuite en accentuant le son [a] dans Ranzen et gepackt dont les occlusives contribuent à l'impression de quelque chose de cassant, de knackig).

\section{Confirmation de l'importance du rythme par les recherches cognitives sur la mémoire}

Les chercheurs en mémoire viennent corroborer l'hypothèse d'une participation du rythme dans cette solution de notre problème de traduction. Ils ont constaté, en effet, que les mots sont stockés dans notre cerveau selon certains principes. On ne sera pas surpris d'apprendre que l'un de ces principes est d'ordre sémantique: «[...] the results of word associations experiments [show] that words are stored in semantic fields and that coordinates are closely associated» (Aitchison 2003: 88).

Les expériences menées par les cognitivistes laissent cependant supposer des réseaux de stockage bien plus complexes. Les observations faites dans le cadre de l'apprentissage de la langue maternelle par les enfants (voir Aitchison 2003: 207) autant que les expériences menées auprès d'aphasiques, ainsi que les tests dans lesquels on a demandé de former des «nonsense words» ont montré que "people try to retain the stress pattern at the base» (Aitchison 2003: 181; notre mise en relief). Ceci se manifeste par le fait que dans les processus d'élicitation, ce ne sont pas seulement les mots sémantiquement proches qui sont appelés simultanément de la mémoire à long terme dans la mémoire active, mais également des éléments linguistiques qui se ressemblent du point de vue du rythme. L'importance du rythme, que le traducteur de textes en prose a souvent l'habitude de négliger, est également soulignée par Risku (voir 1998: 164).

C'est cette ressemblance rythmique qui a été l'élément déclencheur de notre solution créative; solution dans laquelle les relations sémantiques entre les différentes isotopies présentes dans le texte ont évidemment délimité et pour ainsi dire balisé le terrain sémantique à l'intérieur duquel pouvait se développer cette créativité. Si nos deux informateurs en conversation ont pu caractériser le décalage entre la phrase 
française et la première solution proposée - Die Mittagspause ist heilig - comme une opposition entre emotional [émotionnel] et wohlgeformt [bien formé], c'est en raison de la différence de rythme. Celui-ci joue un rôle particulièrement important dans cette dernière phrase qui clôt le texte, ce que les informateurs ont évidemment senti.

Nos corpus nous ont aussi permis de vérifier la pertinence d'une autre observation faite par Aitchison: le rôle joué par la similitude phonétique des mots. "The sound structure is another factor that is taken into account» (Aitchison 2003: 180), lorsqu'il s'agit d'appeler les mots de la mémoire à long terme dans la mémoire active. Jescheniak (2002: 170) introduit le terme de phonologische Koaktivierung [coactivation phonologique] pour désigner ce phénomène. Le fait que nos informateurs du corpus allemand associent spontanément casse-croûte à Sauerkraut [choucroute] montre bien qu'il y a passage au mot français choucroute sous l'effet de la similitude phonétique et qu'il s'agit d'une association spontanée irréfléchie: l'informatrice en question revient, en effet, immédiatement sur sa proposition en se traitant d'idiote et en établissant le rapport avec choucroute. De même, nous avons vu, dans un corpus corse, comment ces associations phoniques peuvent mener à des solutions créatives (voir Thiers 2003: 363-365).

\section{La «pensée latérale» et le changement de focalisation, supports d'une créativité conçue comme une "problem solving activity"}

Un autre facteur déterminant, dans notre corpus, a été l'ouverture à ce que de Bono (1982) appelle la "pensée latérale», par opposition à la pensée logique "verticale» (parallèlement au couple « divergent» et « convergent» chez Guilford 1975). La pensée latérale consiste à adopter une autre perspective par rapport à la chose considérée. Rappelons que la base théorique de cette pensée latérale nous est fournie par la linguistique cognitive, par exemple, d'un Ronald Langacker (1982) et de son figure/ ground alignment, qui dit que nous percevons toujours quelque chose comme proéminent sur un arrière-plan et que cette relation entre arrière-plan et élément proéminent n'est pas fixée une fois pour toutes, mais qu'avec un changement de focalisation, un élément de l'arrière-plan peut devenir proéminent et que l'élément proéminent peut, à son tour, venir se fondre dans l'arrière-plan. Voyons comment ce changement de focalisation s'est opéré dans le cadre de notre texte.

\section{Changement de focalisation à l'intérieur de notre texte}

Dans notre cas, soit la traduction de «l'heure du casse-croûte, c'est sacré» par Wenn es schellt, wird der Ranzen gepackt!, il faut élargir la notion de changement de focalisation à l'ensemble du texte. Avec cette traduction, on assiste, en effet, à un passage d'un cadre - on pourrait aussi parler de "scénario » (Oeser et Seitelberger, 1988:144; Lakoff, 1987) - à un autre: du cadre/scénario "mentalité socialo-prolétaire», on passe au cadre/scénario «mentalité cancre». Voyons le problème qui se pose au traducteur.

Ce qui déclenche ce changement, c'est le «bagage cognitif» (world knowledge) du traducteur bi-culturel, c'est-à-dire le script, le scénario qu'il suppose - que ce soit à juste titre ou non - dans la tête de ce journaliste du Canard enchaîné critique du gouvernement socialiste. Ce scénario s'appelle «mentalité de prolétaire» - qui est l'étiquette à coller sur le front de ces ministres socialistes - qui est étroitement liée à 
l'idée du «manque de responsabilités». L'auteur veut mettre en garde contre un gouvernement composé de prolétaires qui laissent tomber l'outil dès que la sirène, annonçant la pause, se met à hurler, contrairement à un gouvernement de droite qui, conscient de ses responsabilités, ne quitterait pas les lieux avant d'avoir fini de discuter les problèmes (un de nos informateurs/traducteurs travaillant en couple avec une Française l'a bien compris, puisqu'il paraphrase métaphoriquement pour faire comprendre à sa partenaire: lässt den Hammer fallen [laisse tomber le marteau], ne se rendant pas compte qu'en paraphrasant, il avait en fait déjà formulé une proposition de traduction possible; un manque de conscience stratégique que nous avons fréquemment observé dans nos corpus). C'est là le bagage cognitif qui fournit la base du processus top-down qui se déroule dans la tête du traducteur et qui forme le script en fonction duquel il gère l'information du texte.

Ce processus top-down ne vient cependant pas de rien, comme le suggérerait le terme d'«intuition foudroyante» utilisé par Paepcke (Paepcke et Forget, 1981) pour décrire ce genre de traductions créatives. Nihil ex nihilo! Il est étroitement lié à un processus bottom-up qui trouve ses fondements dans le co-texte. Le traducteur a procédé à un enchaînement co-textuel, qui n'est pas sans rappeler le chaining de Lakoff (1987), passant d'une isotopie à une autre, présente dans le texte, l'isotopie fournissant la base linguistique du scénario tel que nous l'avons défini ci-dessus. On a affaire au passage d'un scénario à un autre. Le texte présente, en effet, trois isotopies fondamentales pour la compréhension du texte:

- l'isotopie du Conseil des ministres, avec sa terminologie politique et le style châtié du langage écrit;

- l'isotopie d'une classe de cancres qui ne pense qu'à faire des bêtises; cette isotopie se développe parallèlement à la première; elles sont étroitement entrelacées de sorte que le lecteur procède à une comparaison implicite tout à fait favorable à l'enchấnement associatif des deux isotopies;

- l'isotopie d'une réunion d'ouvriers qui n'attendent que le hurlement de la sirène annonçant la pause.

Ces trois isotopies constituent la base de scénarios. Si on les analyse à travers le figure/ground alignment de Langacker (1987), on peut dire qu'ils se situent tous sur un fond de "comportement irresponsable». C'est là le rhème de ce texte. Or, nous avons vu que des associations entre deux scènes ou scénarios se faisaient sur la base d'un élément commun aux deux. Ici, c'est le fond commun d'un comportement irresponsable qui fournit l'élément commun permettant d'associer le scénario $\mathrm{du}$ «monde socialo-prolétaire» et celui des «cancres inattentifs». En traduisant par l'image d'une classe de cancres dénuée de tout sens des responsabilités, qui n'attend que la sonnerie pour se précipiter dans la pause, sans se soucier le moins du monde de ce que le professeur est en train de dire, ce rhème du manque du sens des responsabilités est rendu de façon optimale dans le texte cible, parce qu'il est tenu compte à la fois des éléments langagiers du texte de départ (l'aspect bottom-up) qui balisent pour ainsi dire la créativité du traducteur, et de l'horizon culturel du destinataire du texte (aspect top-down), en centrant l'attention sur un autre aspect du texte plus approprié à produire le même effet ${ }^{13}$ dans la culture cible: les cancres auxquels sont comparés les ministres ont le même comportement irresponsable dans l'imaginaire des deux cultures en présence dans cette opération traduisante, au contraire de la classe «ouvrière». Il s'agit d'un acte créatif qui résout un problème 
de traduction dans l'esprit d'une créativité conçue comme une problem solving activity (Guilford 1975).

\section{L'explication cognitiviste fournie par les recherches sur la mémoire}

Les chercheurs en mémoire fournissent des modèles explicatifs qui légitiment ce genre de processus associatifs. Roger Schank (1982) nous explique que nos expériences sont stockées dans notre mémoire selon certaines règles. Ainsi il existe, à un niveau d'abstraction assez élevé, ce qu'il appelle les Thematic Organisation Points (TOPs) [points d'organisation thématique] dans lesquels sont stockées toutes les expériences à structure semblable. West Side Story et Roméo et Juliette sont associés sur la base d'un même schéma, d'une même Gestalt: deux amants qui s'aiment face à un entourage qui s'oppose à cet amour; dans West Side Story, c'est le gang, dans Romeo et Juliette, c'est la famille. Dans notre texte, le comportement des représentants de la classe ouvrière et le comportement de la classe scolaire ont une Gestalt similaire et sont associés par le même TOP: l'absence du sens des responsabilités. Écoutons Schank, dans le chapitre 7 de Dynamic Memory intitulé «What is a TOP?»:

A great deal of our ability to understand and of our ability to be creative and novel in our understanding is due to our ability to see connections between events and to draw parallels between events. [...] Thus there must be structures that capture similarities between situations that occur in different domains. (Schank 1982: 110; notre mise en relief)

C'est une relation entre créativité et compétence associative extrêmement significative pour le didacticien de la créativité en traduction, que nous révèlent ici les chercheurs en sciences cognitives.

\section{Avait-on besoin de recourir à cette traduction créative?}

À vrai dire, et si l'on s'en tient au caractère graduable de la créativité dégagé par les chercheurs en créativité, on peut dire qu'il y a déjà un certain degré de créativité dans la traduction par die Mittagspause ist heilig [la pause de midi est sacrée], même si le résultat est quelque peu pâlot. Il ne provient pas, en effet, d'une simple traduction/ reproduction de ce qui se trouve dans le texte français, le bagage cognitif du traducteur et une certaine «manipulation» du texte interviennent pour une grande part.

L'étape die Stunde des Mittagessens par laquelle passent explicitement la plupart des informateurs (et qu'il faut supposer implicite chez les autres) pour aboutir à Mittagspause peut s'interpréter comme un «élargissement scénique» (si nous pouvons nous permettre cette innovation terminologique à partir de la notion de scene), si l'on conçoit avec Fillmore et sa théorie des scenes-and-frames que le processus de compréhension d'un texte passe toujours par la visualisation d'une scène: élargissement scénique de casse-croûte à repas de midi.

Nous passons ensuite, dans une deuxième étape, par un déplacement de focalisation conforme au figure/ground alignment langackerien, de l'objet matériel, à valeur symbolique, casse-croûte, qui constitue la raison matérielle de l'interruption brusque du Conseil des ministres, et qui fait partie de la scène Mittagessen, vers le cadre plus abstrait de la Mittagspause/pause de midi qui, dans le texte de départ constituait l'arrière-plan du symbolique casse-croûte. Nous assistons donc au passage d'un élément scénique au cadre (frame). 
Ce processus a été favorisé par l'étape intermédiaire Mittagessen, qui a introduit la notion de temps, qui conduit, par la suite, à l'association avec pause. Le mot

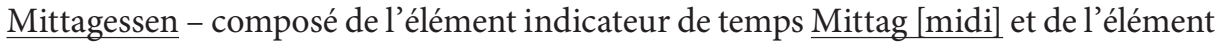
matériel essen [manger], représentant plus abstrait de casse-croûte - est devenu, du fait de la réunion de ces deux éléments dans un seul concept, le mot charnière grâce auquel s'effectue le changement de focalisation de l'aspect matériel du casse-croûte vers l'aspect plus abstrait de la pause. Le passage de Mittagessen à Mittagspause est le passage d'un cadre (frame) à un autre plus vaste et plus abstrait.

Le facteur qui a déterminé ce changement de focalisation est l'environnement co-textuel, dans lequel apparaît de façon massive une isotopie temporelle: douze heures trente pétantes, prolongations, heure (du casse-croûte), c' (comme reprise syntaxique de l'aspect temporel, qui n'en est que d'autant plus accentué).

À côté de ces processus bottom-up, des processus top-down ont également contribué à cette solution traduisante, ce qui est prouvé par l'exclamation de l'informatrice 2 du corpus italien qui s'écrie un peu naïvement indignée: "Ce n'est pas casse-croûte à midi pétantes, ce n'est pas du tout casse-croûte!» (corp. it. 1., 729-730; voir note 11), paraphrasant par midi pétantes le $12 \mathrm{~h} 30$ pétantes du texte. C’est évidemment son bagage cognitif qui lui dit que $12 \mathrm{~h} 30$ est l'heure où d'habitude les gens de l'environnement culturel dont elle fait partie prennent le repas de midi (ce ne serait pas le cas, du moins avec cette précision, dans des pays orientaux). Ceci montre bien l'importance d'une coopération équilibrée entre les deux processus pour arriver à une bonne traduction.

Toutes ces opérations mentales s'expliquent, rappelons-le, par la façon dont sont stockées nos expériences dans notre cerveau et les enchaînements associatifs résultant de la structuration de nos voies neuronales.

\section{La créativité au service de la «Wirkungsgleichheit»}

On peut se demander si cette démarche était nécessaire et si la traduction par Stulle ou par «l'heure du repas est sacrée» n'aurait pas suffi. Elle était nécessaire pour satisfaire autant aux réflexions théoriques des traductologues qui prônent la Wirkungsgleichheit [adéquation de l'effet produit], préconisée par la théorie du skopos de Reiß et Vermeer 1984, qu'à celles des chercheurs en créativité, pour lesquels l'insatisfaction doit déclencher la recherche d'une solution créative. Cette insatisfaction est due au bagage cognitif du traducteur bi-culturel, à son expérience du monde, qui représente le processus top-down en termes cognitivistes, l'«horizon du lecteur» en termes herméneutiques gadamériens. Il sait, en effet:

- qu'en Allemagne le football (associé au mot prolongations) n’a pas la même connotation prolétarienne qu'en France;

- que la société allemande est loin d'imputer le moindre manque du sens des responsabilités à l'ouvrier allemand, au contraire du journaliste du Canard enchaîné, qui sait qu'il peut s'appuyer sur ce cliché qu'il sait vivant dans la mémoire collective des Français (à l'époque de la publication de l'article) et par conséquent du récepteur de son texte;

- et, finalement, qu'il n'existe pas, dans l'univers culturel allemand un équivalent du casse-croûte français (avec lequel on associe en France l'ouvrier du bâtiment prenant son deuxième petit déjeuner pendant la pause, sur le chantier, plutôt qu'un ministre au repas de midi). 
De sorte que le traducteur ne peut pas reprendre, tel quel, le scénario du manque du sens des responsabilités qui sert à caractériser les ministres socialistes dans le texte original français. Il doit recourir à une solution créative. Les chercheurs en créativité, qui considèrent la créativité non pas comme un processus mystérieux au-delà de tout contrôle, mais comme une solution face à la confrontation à un problème (Guilford, 1975) font appel à la "pensée latérale» (de Bono, 1970) ou "pensée divergente» (Guilford, 1975) pour résoudre ce problème. Celle-ci consiste fondamentalement en un changement de focalisation: sur le fond de l'élément commun «manque de sens des responsabilités » l'attention qui était centrée sur le scénario des ministres socialoprolétaires se reporte sur le scénario de la classe des cancres, caractérisée par le même manque du sens des responsabilités, pour arriver ainsi à établir une adéquation de l'effet produit (Wirkungsgleichheit) et à maintenir la fonction du texte, car, contrairement aux ouvriers, les cancres se comportent de la même façon dans les deux cultures en présence et font naître les mêmes associations d'un manque du sens des responsabilités. En termes schankiens: ils sont associés du point de vue du TOP «mise en scène prototypique du manque du sens des responsabilités». Ce changement est induit par toute une mise en scène voulue par l'auteur - la place limitée qui nous est attribuée dans cet article ne nous permet pas d'en discuter tous les détails maintenant -, dont le signe le plus évident est le comportement du président Mitterrand qui prend l'identité d'un maître d'école.

\section{Conclusion}

Cet exemple nous apprend:

- que la créativité n'est pas un processus mystérieux, qui doit être écarté de la réflexion scientifique en traductologie, sous prétexte qu'il n'est pas systématisable; mais

- qu'elle doit être considérée comme une problem solving activity, comme un outil du traducteur, lui permettant de résoudre les problèmes posés par les barrières culturelles;

- que pour satisfaire aux critères de scientificité, établis par Karl Popper (1935), le traducteur doit être capable de procéder à une reconstruction du chemin parcouru pour arriver à la solution créative d'un problème;

- qu’en traductologie, le critère poppérien de créativité est assuré par la «traçabilité intersubjective», qui consiste à rendre transparent le processus de la construction du sens;

- que la pertinence d'une théorie de la traduction se mesure à sa capacité d'intégrer tous les aspects que nous venons de citer, afin d'être reconnue comme utile par les praticiens, qui, eux, se heurtent à l'incontournable subjectivité en traduction (voir Cercel, 2013: 301-342);

- que cette incontournable subjectivité en traduction a depuis toujours été prise en considération par l'approche herméneutique - le fameux «cercle herméneutique» (Heidegger, 1927) -, sans que cette approche ne se soit jamais laissé dérouter par les considérations structuralistes des traductologues «objectivistes»;

- que cette approche herméneutique se voit soutenue par les résultats récents obtenus par les chercheurs en sciences cognitives, dont les fameux processus bottom-up et 
top-down (Lakoff, 1987) ne sont autre chose que les éléments intégrés dans la fusion des horizons (Horizontverschmelzung) de Gadamer (1960);

- qu'une didactique de la traduction qui prétend former des praticiens se doit d'intégrer l'étude de la créativité dans ses fondements théoriques ainsi que tous les aspects qui y affèrent, notamment la connaissance de certains aspects du fonctionnement de notre cerveau qui permettront au traducteur non seulement d'assumer sa créativité intuitive sans le sentiment de culpabilité qu'elle risque d'engendrer auprès des esprits mal préparés (Thiers, 2003; Bălăcescu et Stefanink, 2003), mais aussi d'apprendre à développer les stratégies destinées à créer des situations propices à l'éclosion de la créativité, comme nous y invitent les chercheurs en créativité;

- que cette didactique doit s'appuyer sur une méthode de recherches proche de la réalité traductive qui observe la construction du sens in vivo, comme c'est le cas de l'«ethnotraductologie» (Stefanink, 1995) qui met en œuvre les principes de l'analyse ethnométhodologique conversationnelle;

- que cette analyse doit s'appuyer sur ce que les neurophysiologistes nous apprennent quant à la constitution et la dynamique des réseaux neuronaux, notamment le développement et l'évaluation de la compétence associative du traducteur.

\section{NOTES}

1. «Ressemblance familiale» (notre traduction).

2. Littéralement «traces dans la mémoire».

3. Lenk (2014: 78) parle d'un Einschwingprozeß von entsprechenden Neuronenensembles: "worauf sich dann benachbarte Neuronen bzw. Neuronen, die sich in einem zu bildenden Netzwerk «zusammenfinden», auf ein und dieselbe Schwingungsrate einschwingen - also nun kohärent oszillieren».

4. «Imprägnieren» in diesem Sinne ist also eigentlich etwas, was den Beitrag der Welt verbindet mit einem strukturierenden Verfahren, in das wir selber « eintreten »; und zwar ist beides ineinandergeschachtelt (Lenk, 2014: 81).

5. Selon Thiers (2003), qui utilise exactement ces mots pour décrire comment il aborde la traduction d'un texte, faisant innocemment de la philosophie gadamérienne, comme Monsieur Jourdain faisait de la prose, sans le savoir.

6. Nachvollziehbarkeit fait partie de tout un ensemble de termes qui, dans l'approche herméneutique, sont caractéristiques d'une attitude dynamique dans l'approche du sens.

7. Le mercredi, à l’Élysée. Le Canard enchaîné. 13 avril 1983, numéro 3259.

8. En tout cas, ceci vaut pour l'époque où le texte a été publié, donc avant la Coupe du monde de football de 1998 en France, à l'occasion de laquelle le président de la République a bien voulu enfiler un maillot de football pour faire spectacle. Mais à en croire notre enquête actuelle, cet événement n'a guère fait changer les choses.

9. Ce qui peut paraître paradoxal face à l'idée fort répandue d'une créativité qui viendrait spontanément, «comme l'enfant à la Vierge», par hasard, mais se situe tout à fait dans l'esprit d'une créativité conçue comme une "problem solving activity». Pour ce qui est de la démystification de la créativité dans la création littéraire, Poe (1846) en fournit un exemple frappant dans sa description du cheminement qui conduit à la création de son fameux poème The Raven.

10. Notons que dans son dernier livre, Gerzymisch (2013) effectue une volte-face pour adopter une position plus ouverte à l'intuition, suivant ainsi une tendance évolutive actuelle vers une reconnaissance de l'approche herméneutique en traduction, de sorte que nous avons été amenés à parler du «tournant herméneutique» dans la pensée de Gerzymisch (Stefanink et Bălăcescu, 2014).

11. Corpus pris sur le vif avec nos étudiants de Bielefeld, que nous ne reproduisons pas par manque d'espace. Nous avons ainsi un nombre de corpus enregistrés (entre 1983 et 2004) selon la même méthode à titre comparatif; qu'il nous soit permis de recourir à eux sous forme de «corp. it.»= corpus italien, «corp. all.» = corpus allemand. Le corpus italien a été enregistré dans le cadre d'une formation de formateurs de professeurs de FLE que nous avons assurée dans l'un des stages d'été 
du CREDIF (au cours des années 1980), le corpus allemand dans le cadre de nos cours à l'Université de Bielefeld. Une étude comparative approfondie, comme nous la pratiquons dans nos stages de formation, est d'une valeur didactique inestimable.

12. Elle est germaniste roumaine et le mot Ranzen lui est inconnu.

13. En conformité avec le critère de la Wirkungsgleichheit [adéquation de l'effet produit] déterminant l'évaluation de la qualité d'une traduction selon la théorie du skopos [Skopostheorie] (Reiß et Vermeer, 1984).

\section{RÉFÉRENCES}

Aitchison, Jean (2003): Words in the Mind. An Introduction to the Mental Lexicon, Oxford: Blackwell.

BĂLĂCESCU, Ioana et STEFANINK, Bernd (2003): Une approche théorique pour la traduction poétique. In: Ghjacumu THIERs, dir. Baratti. Un échange de commentaires sur la traduction de la poésie. Ajaccio: Albian, 24-77.

BĂLĂCESCU, Ioana et STEFANINK, Bernd (2008): Une didactique de la créativité en traduction, conçue comme "problem solving activity». Studia Universitatis Babeș-Bolyai Philologia. 53(3):36-51.

CerCEL, Larisa (2013): Übersetzungshermeneutik. Historische und systematische Grundlegung. Saint-Ingbert: Röhrig.

De Bono, Edward (1970): Lateral Thinking. A Textbook of Creativity. Londres: Ward Lock Educational.

Fillmore, Charles (1976: Scenes-and-Frames Semantics. In: Antonio Zampolli, dir. Linguistic Structures Processing, Amsterdam: N. Holland, 55-88.

GAdAmer, Hans-Georg (1960): Wahrheit und Methode. Grundzüge einer philosophischen Hermeneutik, Tübingen: J.C.B. Mohr.

GARFinkel, Harold (1984): Studies in Ethnomethodology. Cambridge: Blackwell.

Gerzymisch, Heidrun (2013): Translation als Sinngebung. Münster: LitVerlag.

Gerzymisch-Arbogast, Heidrun, Mudersbach, Klaus (1998): Methoden des wissenschaftlichen Übersetzens. Tübingen: Francke.

Guilford, Joy Peter (1975): Creativity: A Quarter Century of Progress. In: Irving A. TAYLOR, Jacob W. Getzels, dir. Perspectives in Creativity, Chicago: Aldine, 37-59.

Heidegger, Martin (1927/1993): Sein und Zeit, Tübingen: Niemeyer.

Hermans, Theo, dir. (1985): The Manipulation of Literature: Studies in Literary Translation. Londres: Croom Helm.

HönIG, Hans (1989): Die übersetzerrelevante Textanalyse. In: Franck KöNIGs, dir. Übersetzungswissenschaft und Fremdspracheunterricht. Munich: Goethe-Institut, 121-145.

Ionescu, Tudor (2003) : Știința sau/și arta traducerii [La science ou/et l'art de la traduction]. Cluj-Napoca: Editura Limes.

Jescheniak, Jörg D. (2002): Sprachproduktion. Der Zugriff auf das lexikale Gedächtnis beim Sprechen. Göttingen/Bern/Toronto/Seattle: Hogrefe.

KRINGS, Hans Peter (1986): Was in den Köpfen von Übersetzern vorgeht. Eine empirische Untersuchung zur Struktur des Übersetzungsprozesses an fortgeschrittenen Französischlernern. Tübingen: Narr.

Lakoff, George (1987): Women, Fire and Dangerous Things. What Categories Reveal about the Mind. Chicago: University of Chicago Press.

LakofF, George et Johnson, Mark (1980): Metaphors We Live By. Chicago: University of Chicago Press.

LANGACKer, Ronald W. (1987): Foundations of Cognitive Grammar. Stanford: Stanford University Press.

LENK, Hans (2014): Methodologischer Schemainterpretationismus: Erkenntnistheoretisches zur Einführung. In: Gregor Paul. Transkulturelle Logik. Universalität in der Vielfalt. Bochum/ Fribourg:Projektverlag, 71-98. 
Mavrodin, Irina (1982/1998): Poietică și Poetică [Poïétique et Poétique]. Craiova: Scrisul Românesc.

Mavrodin, Irina (2001): Cvadratura Cercului [La quadrature du cercle]. Bucarest: Editura Eminescu.

Oeser, Erhard et Seitelberger, Franz (1988): Gehirn, Bewußtsein und Erkenntnis. Darmstad: Wissenschaftliche Buchgesellschaft.

PAepcke, Fritz et Forget, Philippe (1981): Textverstehen und Übersetzen - Ouvertures sur la traduction. Heidelberg: Groos.

PoE, Edgar Allan (1846): The Philosophy of Composition. Graham's American Monthly Magazine of Literature and Art. Consulté le 12 octobre 2014, http://xroads.virginia.edu/ HYPER/poe/ composition.html

Popper, Karl R. (1935): Logik der Forschung. Vienne: Springer.

Pritzel, Monika, Brand, Matthias et Markowitsch, Hans J. (2003): Gehirn und Verhalten. Berlin: Spektrum Akademischer Verlag.

ReIss, Katharina et VERMEer, Hans (1984): Grundlegung einer allgemeinen Translationstheorie, Tübingen: Niemeyer.

Risku, Hanna (1998): Translatorische Kompetenz: kognitive Grundlagen des Übersetzens als Expertentätigkeit. Tübingen: Stauffenburg.

Rosch, Eleanor (1973): Natural categories. Cognitive Psychology. 4:328-350.

Schade, Ulrich (1992): Konnektionismus. Zur Modellierung der Sprachproduktion. Opladen: Westdeutscher Verlag.

Schank, Roger C. (1982): Dynamic Memory. A Theory of Reminding and Learning in Computers and People. Londres/New York: Cambridge University Press.

Stefanink, Bernd (1991): Vom Nutzen und der Notwendigkeit der Theorie für den universitären Übersetzungsunterricht. In: Bla asch, Hans-Werner, Сhaix, Paul et Malamah-Thомas, Ann, dir. The role of translation in foreign language teaching / Die Rolle der Übersetzung im Fremdsprachenunterricht / Le rôle de la traduction dans l'enseignement des langues étrangères. Paris: Didier Érudition, 59-84.

Stefanink, Bernd (1995): L'ethnotraductologie au service d'un enseignement de la traduction centré sur l'apprenant. Le langage et l'homme. 4:265-293.

Stefanink, Bernd (1997): «Esprit de finesse» - «Esprit de géométrie» Das Verhältnis von «Intuition» und 'übersetzerrelevanter Textanalyse' beim Übersetzen. In: Rudi KeLLER, dir. Linguistik und Literaturübersetzen. Tübingen: Narr, 161-184.

Stefanink, Bernd et BăLăCESCU, Ioana (2014): Compte rendu de Heidrun Gerzymisch (2013): Translation als Sinngebung. Münster: LitVerlag. Lebende Sprachen. 59(1):188-207.

STOLze, Radegundis (2003): Hermeneutik und Translation. Tübingen: Narr.

Thiers, Ghjacumu (2003): L'écart parfait. In: Ghjacumu Thiers, dir. Baratti. Un échange de commentaires sur la traduction de la poésie. Ajaccio: Albian, 363-372.

\section{ANNEXE}

\section{Corpus créatif}

A was ich nicht so richtig in den Griff kriege ist das c ... also praktisch das Demonstrativpronomen, das den vorherigen Satzteil noch einmal zusammenfasst ... da ist irgenwie ein Stilbruch drin ... das ist gesprochene Sprache ... Umgangssprache ... das ist irgenwie emotional .... während der deutsche Satz eine wohlformulierte Information wiederzugeben scheint ... da ist die ganze Emotionalität raus ...

B stimmt...

A ... l'heure du casse-croûte c'est sacré klingt irgendwie stärker ... das haut so richtig rein ... als Satz am Schluss ... das ist bei die Mittagspause ist heilig nicht so drin

B stimmt, da hast du Recht ... aber wie willst du das sonst sagen ... mir fällt da nichts ein ... die Stunde des Sandwich ... im Rumänischen könnte man casse-croûte als Sandwich übersetzen

A ja aber die Stunde des Sandwich klingt ja wirklich komisch ... ich denke dann gleich Wem die Stunde schlägt ...

B wieso Wem die Stunde schlägt? 
A na ja, Hemingway For whom the Bell tolls ... das ist die deutsche Übersetzung ... ich sehe dann gleich Szenen aus dem spanischen Bürgerkrieg, wie sie von Hemingway geschildert werden ... wie sie sich da den Berg hinaufkämpfen und beschossen werden ... nein ... wir müssen uns einfach vorstellen wie die Typen da in unserem Text herumsitzen ... so dahingefletzt ...

B dahingefletzt?... Was heißt das?

A na ja ... ich meine... so ohne richtige Haltung ... ohne richtiges Interesse ... gelangweilt von dem was der Lehrer da oben herumstottert ... total desinteressiert ... sie warten nur noch auf die Mittagspause ... wie die gelangweilten Schüler in der Klasse ... die hören ja nicht zu ... die warten doch nur dass es schellt ... und dann rennen sie in die Pause ...

B $\quad \ldots$ ja $\ldots$ stimmt ...

A ... wenn es schellt wird der Ranzen gepackt!... genau das ist es ...

B oh, ja ... super ... das klingt gut ... aber was heißt genau Ranzen?

A ja ... das ist eben der Schulranzen ... schon etwas veraltet, aber irgendwie packt man eben den Ranzen und nicht etwa die Schultasche ... klingt eben knackiger ...

B stimmt...

A ... das hat denselben ... entschiedenen Charakter wie l'heure du casse-croûte c'est sacré ... und da es auch noch der letzte Satz im Text ist, der sozusagen den Schlusstrich zieht, ist es wichtig so einen knackigen Abschluss zu haben ... wie im Französischen ...

B $\mathrm{hmm} \ldots$ (zustimmend)

A man hat das Gefühl, dass es ein abschließender Satz ist, der alles sagt, was zu sagen ist und das Tableau so zusagen abrundet ... abschließt ... da gibt es nichts mehr hinzuzufügen oder zu entgegnen ...

B hmhm (zustimmend). 